\title{
Using Bifactor EFA, Bifactor CFA and Exploratory Structural Equation Modeling to Validate Factor Structure of the Meaning in Life Questionnaire, Greek Version
}

\author{
Anastasios Stalikas, Theodoros A. Kyriazos, Vasiliki Yotsidi, Konstantina Prassa \\ Department of Psychology, Panteion University, Athens, Greece \\ Email: th.kyriazos@gmail.com
}

How to cite this paper: Stalikas, A., Kyriazos, T. A., Yotsidi, V., \& Prassa, K. (2018). Using Bifactor EFA, Bifactor CFA and Exploratory Structural Equation Modeling to Validate Factor Structure of the Meaning in Life Questionnaire, Greek Version. Psychology, 9, 348-371.

https://doi.org/10.4236/psych.2018.93022

Received: February 13, 2018

Accepted: March 24, 2018

Published: March 27, 2018

Copyright $\odot 2018$ by authors and Scientific Research Publishing Inc. This work is licensed under the Creative Commons Attribution International License (CC BY 4.0).

http://creativecommons.org/licenses/by/4.0/

\begin{abstract}
The purpose of this study was to evaluate the structure, invariance, reliability, convergent and discriminant validity of the MLQ with exploratory and confirmatory factor analysis in 1561 Greek adults. After sample-splitting, a two-factor EFA and a two-factor bifactor EFA model were tested in the first sample. Then, seven CFA models were tested (three ICM-CFA models, two bifactor CFA models and two ESEM models) in the second sample. Two-factor ESEM representation was notably better than the ICM-CFA models and marginally better than the bifactor model. Optimal ESEM model was successfully cross-validated in a third sample. MLQ was also proved to be invariant across gender. Internal consistency reliability was adequate. Convergent and discriminant validity were also examined. Considering all findings, MLQ is gender invariant, reliable and valid to use in Greek cultural context.
\end{abstract}

\section{Keywords}

MLQ Factor Structure, ESEM, ICM-CFA, Bifactor EFA, Bifactor CFA, Measurement Invariance, Greece

\section{Introduction}

In recent years, the construct of meaning in life has been extensively studied by a plethora of social scientists, concertedly with the emphasis that has been put on several other variables of positive psychology (Ryan \& Deci, 2001; Seligman \& Csikszentmihalyi, 2000). The meaning in life concerns the degree to which people think their life matters or has a purpose (Steger \& Kashdan, 2006) and is 
a prominent indicator of well-being (Ryff, 1989; Steger \& Frazier, 2005). The pervasive relation between meaning in life and well-being has been consistently documented across several studies (e.g., Reker, Peacock, \& Wong, 1987; Steger \& Frazier, 2005). Those who perceive their life as meaningful display higher optimism and self-actualization levels (Compton, Smith, Cornish, \& Qualls, 1996), self-esteem (Steger et al., 2006) and positive affect (King, Hicks, Krull, \& Del Gaiso, 2006). On the other hand, the failure to achieve meaning in life may lead to depression and anxiety (Steger et al., 2006; Debats, van der Lubbe, \& Wezeman, 1993), substance abuse (Steger et al., 2008a; Steger \& Kashdan, 2006), increased suicidal ideation (Harlow, Newcomb, \& Bentler, 1986) and other mental health problems, mainly due to the experience of boredom, emptiness and apathy (Frankl, 1963).

Following several decades of empirical and theoretical work on the significance of meaning in life (e.g., Frankl, 1963; Ryff \& Singer, 1998; Steger, 2012), two important dimensions of the concept have been emerged (e.g., Crumbaugh, 1977; Steger et al., 2006): 1) presence of meaning and 2) search for meaning. Presence of meaning in life refers to the extent to which people perceive their life as comprehensible, important and meaningful (Steger \& Kashdan, 2006) and is recognized as a construct that leverages positive functioning (Steger \& Shin, 2010). Individuals who feel that their life is meaningful experience greater well-being and lower levels of psychological distress (Steger et al., 2006). Moreover, they have smoother adjustment after experiencing post-traumatic events (Steger et al., 2008b) and lower levels of neuroticism (Zika \& Chamberlain, 1992). They report better health (Steger, Mann, Michels, \& Cooper, 2009) and have reduced likelihood of cognitive decline (Boyle, Buchman, Barnes, \& Bennett, 2010).

The second dimension, search for meaning in life, concerns the need for intently seeking a valued life purpose or mission and a greater meaning in life and originates from human intrinsic motivation (Frankl, 1963). Although search for meaning has been found to result to resilient responses in cases of people facing negative events (e.g., Skaggs \& Barron, 2006; Lee, 2008; Chan \& Chan, 2011), in non-stressful situations it is linked to higher levels of depression and anxiety (Steger et al., 2009), negative affect (Steger et al., 2008b) and reduced levels of happiness and life satisfaction (Park, Park \& Peterson, 2010). In most of the studies conducted (e.g., Steger et al., 2006; Damasio \& Koller, 2015; Boyraz, Lightsey Jr., \& Can, 2013; Temane, Khumalo, \& Wissing, 2014), negative correlations have been documented between the two dimensions. This can be attributed to the fact that perceived meaning is associated with less of a need to pursue further meaning in life (Schulenberg, Strack, \& Buchanan, 2011). Nevertheless, it is noticeable that in some studies this result is overturned and the relationship between them has been reported to be positive, especially in collectivistic cultures such as China and Japan (Steger et al., 2008c; Wang \& Dai, 2008). This finding may result from the differences in social and value orientations 
(Steger et al., 2008c). As the interest towards the understanding of human well-being continuously grows (Seligman, Steen, Park, \& Peterson, 2005; Seligman \& Csikszentmihalyi, 2000), the necessity of using psychometrically sound research tools and rigorously validated measures for assessing such constructs has become imperative (Diener \& Seligman, 2004). Meaning in life is one of the most critical components to provide the conditions from which happiness arises (Lent, 2004; Ryff \& Singer, 1998) and thus the creation of psychometric scales for its measurement has been deemed indispensable. Several measures of meaning in life have been developed in previous work, such as the Purpose in Life Test (PIL; Crumbaugh \& Maholick, 1964), the Life Regard Index (LRI; Battista \& Almond, 1973) and the Sense of Coherence Scale (Antonovsky, 1987). However, these scales have been criticized for being confounded with several of the variables they correlate with (Frazier, Oishi, \& Steger, 2003) as well as for having poor content validity (Dyck, 1987) and structural properties (Steger, 2007). In an effort to overcome these pitfalls and provide a psychometrically robust measure of the presence of and search for meaning in life, Steger et al. (2006) developed the Meaning in Life Questionnaire (MLQ). MLQ scores have greater stability and better discriminant validity than scores of other scales. In a review of fifty-nine instruments assessing meaning in life, Brandstätter et al. (2012) concluded that MLQ is one of the best scales in terms of concept definition, sampling, item development and forms of analysis.

The Meaning in Life Questionnaire is a self-report inventory that assesses two dimensions of meaning in life. The Presence of Meaning subscale comprises 5 items (items 1, 4, 5, 6, and 9) and measures respondents' perceived meaning in their life while the Search for Meaning subscale also consists of 5 items (items 2, $3,7,8$, and 10) and measures their motivation to discover meaning in their life. The questionnaire takes about 3 - 5 minutes to complete. The MLQ does not have cut scores but it is intended to measure meaning in life across the full range of human functioning.

Steger et al. (2006) reported internal consistency reliability coefficients (Cronbach's alpha) ranging between .81 and .86 for Presence and between .84 and .92 for Search subscale. One-month test-retest reliability coefficients were .70 for Presence and .73 for Search. Other studies also provide support for the reliability and test-retest reliability of MLQ scores. Currently, the instrument has been translated into more than 20 languages for international use and has been validated in several cultures including Greece (Pezirkianidis et al., 2016), Brazil (Damasio \& Koller, 2015), Chile (Steger \& Samman, 2012), Hong Kong (Chan, 2014), Turkey and USA (Boyraz et al., 2013), China (Jiang, Bai, \& Xue, 2016), Japan (Steger et al., 2008c), and South Africa (Temane et al., 2014). During the last few years, the MLQ has also been used in diverse and special populations within the United States, such as individuals experiencing grief (Boyraz \& Efstathiou, 2011; Boyraz, Horne, \& Sayger, 2010), patients suffering from serious mental illnesses (Schulenberg et al., 2011), smoking cessation patients (Steger et 
al., 2009), and individuals from different ethnic backgrounds (Kiang \& Fuligni, 2010). As far as differentiation in demographics variables are concerned, no significant gender differences have been found, while older participants report higher scores in Presence subscale and lower scores in Search subscale (Steger et al., 2006).

The purpose of this study is to validate the MLQ in the Greek context and explore its psychometric properties among non-clinical Greek population. More specifically, the objectives of this study are the following: 1) To validate the construct validity of the Meaning in Life Questionnaire (Steger et al., 2006), Greek Version using both exploratory and confirmatory factorial analysis techniques like Bifactor EFA, Bifactor CFA and ESEM; 2) to examine measurement invariance of MLQ across gender; 3) to study the internal consistency reliability of the MLQ; and 4) to evaluate the convergent and discriminant validity of the MLQ with the constructs of well-being, hope, anxiety, depression, stress, hope and resilience.

\section{Method}

\subsection{Participants}

The sample is a subset of a larger dataset. The data used in this study were collected from 2016 onwards. The sample comprised 1561 Greek adults of the non-clinical population. The participants were on average 39.7 years of age ( $S D=$ 12.81 ) and mostly female (62\%). Most of them were married (51\%), followed by single (41\%), divorced (5\%), widowed (2\%), and $1 \%$ endorsing "other".

\subsection{Materials}

\subsubsection{Meaning in Life Questionnaire (MLQ)}

The MLQ (Steger et al., 2006) is a ten-item measure of perceived meaning and purpose in life. More specifically, it is a two-dimensional scale with five items in each factor. The first factor (Presence of Meaning) includes items about the perceived existence of meaning (e.g., "I have a good sense of what makes my life meaningful"). The second factor (Search for Meaning) examines one's perceived quest for purpose (e.g., "I am always looking to find my life's purpose"). Items are rated on a 7-point Likert scale (from "Absolutely True" to "Absolutely Untrue"). Possible factor scores range from 5 to 35. Scores higher than 24 in each factor show high Presence/Search for meaning. From the scores of the two factors combined, four groups of respondents (Steger, 2010) are possible. The two factors are considered related but distinct. Higher scores suggest greater Presence/Search of meaning. Item nine ("My life has no clear purpose") is reverse-scored.

\subsubsection{Satisfaction with Life Scale (SWLS)}

The Satisfaction with Life Scale (Diener, Emmons, Larsen, \& Griffin, 1985) is a five-item measure of perceived life satisfaction. It contains five items about cognitive appraisals of one's life (e.g., "The conditions of my life are excellent"), 
rated on a 7 -point Likert scale, from $1=$ strongly disagree to $7=$ strongly agree. The higher the score the greater the perceived satisfaction. The range of possible scores is from least satisfaction with life (5) to highest life satisfaction (35). Most of the people fall between 23 - 28 (Pavot \& Diener, 1993). The SWLS has been used both in community samples (c.f. Pavot \& Diener, 2008) and clinical samples (Arrindell, Meeuwesen, \& Huyse, 1991). Internal consistency reliability (Cronbach's alpha) was satisfactory ranging from .79 to .89 (Pavot \& Diener, 1993; Adler \& Fagley, 2005; Steger, et al., 2006; Alfonso \& Allison, 1992a). The SWLS is negatively correlated with depression (Blais, Vallerand, Pelletier, \& Briere, 1989) and with NA (Larsen, Diener, \& Emmons, 1985).

\subsubsection{Subjective Happiness Scale (SHS)}

The SHS (Lyubomirsky \& Lepper, 1999) is a brief and widely used self-report measure of the degree to which the respondent feels happy. Four items are rated on a 7 -point Likert scale (from $1=$ not a very happy person to $7=$ very happy person). Higher scores suggest higher mean happiness. Lyubomirsky and Lepper reported internal consistency reliability ranging from .79 to $.94(M=.86)$. In the same study, test-retest reliability ranged from .55 to .90 . Finally, convergent validity with well-being measures varied from .52 to .72 .

\subsubsection{Trait Hope Scale (HS)}

Trait Hope Scale (Snyder et al., 1991) is a 12-item, self-report measure of dispositional hope (e.g., "I can think of many ways to get out of a jam"). Items are tapping on two factors: Agency and Pathways (or confidence and ability to pursuit goals, respectively). Agency and Pathways represent aspects of hope, distinctly related (Bronk et al., 2009). Responses are rated using an 8-point Likert scale, from 1 (Definitely False) to 8 (Definitely True), such that scores can range from a low of 8 to a high of 64, since four items are fillers. Generally, higher scores suggest a greater sense of hope. In the older version of the HS (Snyder et al., 1991), responses were rated on a four-point Likert scale ( 1 = Definitely False, $4=$ Definitely True). Snyder et al. (1991) reported that for the total scale, Cronbach's alphas varied from .74 to .84 .

\subsubsection{Depression Anxiety Stress Scale (DASS)}

DASS (Lovibond \& Lovibond, 1995) measures emotional distress in three 7-item factors: namely depression (e.g., "I couldn't seem to experience any positive feeling at all"), anxiety (e.g., "I was aware of dryness of my mouth") and stress (e.g., "I found it hard to wind down"). The 21 items are rated on a four-point Likert scale assessing intensity/frequency of distress (from $0=$ did not apply to me at all to 3 = applied to me very much, or most of the time) over the past week. The higher the score the more intense or frequent the emotional distress. Each factor has a distinct score ranging from $0-21$. Scores greater than 14, 10 and 17 suggest extremely severe Depression, Anxiety and Stress respectively (Lovibond \& Lovibond, 1995). Internal consistency reliability was reported $\alpha$ 
$=.97$ for adults of the general population (Henry \& Crawford, 2005), and for each factor alphas ranged between.81 and .97 (McDowell, 2006 cited in Yusoff, 2013).

\subsubsection{The Connor-Davidson Resilience Scale (CD-RISC)}

The Connor-Davidson Resilience Scale (CD-RISC, Connor \& Davidson, 2003) includes 25 items measuring psychological resilience (e.g., Can handle unpleasant feelings). Items are rated on a 5-point Likert scale, from "not true at alP" (0), to "true nearly all of the time" (4). The perceived emotional states are rated over the past month. The possible score varies from 0 to 100 . Higher scores suggest greater resilience. Scores higher than 92 suggest high-resilience individuals. Connor \& Davidson (2003) reported a Cronbach's alpha of .89 for the entire scale. The CD-RISC was primarily developed to measure stress-coping ability. Therefore, it is negatively correlated with perceived stress and positively correlated with social support (Connor \& Davidson, 2003).

\subsection{Procedure}

Data were collected with the help of psychology students who voluntarily administered the test battery to 15 adult persons of their social environment. About 100 students participated in the study receiving extra credit. All participants were voluntarily recruited by the students on the condition they were older than 18 years. A letter was included in the test battery to inform participants about the purpose of this study. More specifically, the following process took place. First, students received a training course on the administration of psychology questionnaires by the research-team members. Then, a period of pilot-testing the battery followed to track any ambiguities. During pilot testing, the time needed to complete the test battery was estimated (approximately 20 minutes). Finally, each student was supplied with 15 copies of the test battery in paper and pencil form to administer them to adults in their social environment individually.

\subsection{Design of the Research}

The sample was split in three parts to study construct validity of MLQ in different samples. More specifically, all analyses were carried out on two levels: 1) on three sub-samples (EFA, CFA1 and CFA2) to examine construct validity and cross-validate it; 2 ) on the entire sample (Total sample), to evaluate measurement invariance across gender, internal consistency reliability and convergent/discriminant validity. In the first sample (EFA Sample), Exploratory Factor Analysis and Bifactor Exploratory Factor Analysis were carried out. Independent Cluster Model Confirmatory Factor Analysis (ICM-CFA), Bifactor Confirmatory Factor Analysis and Exploratory Structural Equation Modeling Analysis followed in the second sample (CFA1 Sample), testing seven alternative solutions. The third sample was used for cross-validation of the optimal CFA model established from the second sample (CFA2 Sample). Then, a multi-group CFA 
(MGCFA) was carried out in the entire sample $(N=1561)$ to test for the measurement invariance of the MLQ across gender. Reliability analysis followed in the entire sample to examine internal consistency. Finally, the relation of MLQ to well-being (namely to life satisfaction and subjective happiness), trait hope, depression, anxiety, stress, and resilience were examined in the total sample. Data collected were coded and analyzed with SPSS Version 25.0 (IBM, 2017), Stata Version 14.2 (StataCorp, 2015) and MPlus Version 7.0 (Muthen \& Muthen, 2012).

\section{Results}

\subsection{Data Screening}

The total sample comprised $N=1561$ cases. Missing values in all variables did not exceed $2 \%$. Missing data analysis followed to examine whether values were missing completely at random (MCAR). Little's MCAR test (Little, 1988) was not significant, Chi-Square $(14,972, N=1561)=15,128.87, p=.182$, suggesting that values were missing entirely at chance. Thus, missing values in the dataset were estimated with the Expectation-Maximization algorithm (EM). This method assumes a distribution for the missing values and makes likelihood-based inferences under that distribution (IBM, 2016). Then EM is calculating a matrix of means and covariances to estimate the missing values (Soley-Bori, 2013).

To validate the MLQ factor structure, the total sample $(N=1561)$ was randomly split into three parts $\left(N_{\mathrm{EFA}}=313, N_{C F A 1}=624, N_{C F A 2}=624\right)$ with the random number generator algorithm of SPSS version 25 (IBM, 2017). Caution was taken to keep enough sample power in all three subsamples (EFA, CFA1, CFA2). A sample-to-variable ratio of 10:1 (Osborne \& Costello, 2005; Singh et al., 2016) or alternately more than 300 cases are generally considered adequate for factor analysis (Tabachnick \& Fidell 1996; Comrey \& Lee, 1992). Our sample-to-variable ratio for the EFA sample $(N=313)$, CFA1 sample $(N=624)$ and CFA2 sample $(N=624)$ was 31.3 and 62.4 respectively. Sample-splitting (Guadagnoli \& Velicer, 1988; MacCallum, Browne \& Sugawara, 1996) is generally a cross-validation method (Byrne, 2010; Brown, 2015).

\subsection{Univariate and Multivariate Normality}

The data in all four samples (Total, EFA, CFA1 and CFA2) violated the assumptions of univariate normality. Specially, Kolomogorov-Smirnov tests (Massey, 1951) on each item of the MLQ were statistically significant for all 10 items $(p<.001)$. Four tests were used to examine multivariate normality: 1) Mardia's multivariate kurtosis test (Mardia, 1970); 2) Mardia's multivariate skewness test (Mardia, 1970); 3) Henze-Zirkler's consistent test (Henze \& Zirkler, 1990), and 4) Doornik-Hansen omnibus test (Doornik \& Hansen, 2008). All four tests rejected the null hypothesis (all $p<.0001$ ), suggesting a violation of multivariate normality of the MLQ scores for all three samples. 


\subsection{Establishing Construct Validity with Exploratory Factor Analysis (EFA)}

For both EFA and CFA, MPlus (Muthen \& Muthen, 2012) uses robust rescaling-based estimators (like robust MLR). MLR is appropriate for non-normal distributions, estimating standard errors and chi-square test statistics. Finally, MLR can handle small to medium-sized samples (Bentler \& Yuan, 1999; Muthen \& Asparouhov, 2002 as quoted in Wang \& Wang, 2012). Considering all the above properties, the robust MLR was used as an estimator for EFA and CFA.

Two exploratory factor analyses were executed in the EFA sample $(N=313)$ : 1) a standard EFA, and 2) a Bifactor EFA (Schmid \& Leiman, 1957). The MLR was used for parameter estimates with Geomin factor rotation. Bi-Geomin factor rotation was used for the Bifactor EFA. A standard EFA was carried out to establish a factor structure and have a baseline model for comparison with the EFA Bifactor model tested subsequently. Generally, relying on an EFA measurement model is usually a prerequisite to examine construct-relevant multidimensionality (Morin et al., 2016a, cited in Howard, et al., 2016). Regarding Bi-factor analysis, Reise et al. (2007) recommended a bifactor always to be tested when checking dimensionality of a construct (cited in Hammer \& Toland, 2016). The Schmid-Leiman method (Schmid \& Leiman, 1957) has been used to test bi-factor CFA models. Nevertheless, Jennrich \& Bentler (2011) recently introduced the Exploratory Bi-factor Analysis method used in this study. EFA model fit was evaluated by the following criteria ( $\mathrm{Hu} \&$ Bentler, 1999; Brown, 2015): RMSEA ( $\leq .06,90 \% \mathrm{CI} \leq .06)$, SRMR ( $\leq .08)$, CFI $(\geq .95)$, TLI $(\geq .95)$, and the chi-square/df ratio less than 3 (Kline, 2016). Fit statistics (see Table 1) suggested that both models achieved acceptable fit to the data with CFI and TLI > .95 and SRMR slightly better for the Bifactor solution. Both Chi-square/df ratios were < 3 , indicating adequate fit to the data (Kline, 2016).

Table 2 contains Geominand bi-Geominfactor loading for both EFA solutions tested. Factor Loadings for simple EFA ranged for the Presence factor from .493 (item 9) to .833 (item 4) and for the Search factor from .633 (Item 10) to .824 (Item 3). All items had significant primary factor loadings to the intended factor with no cross-loading items. Geominfactor correlation between Presence Factor

Table 1. Model fit statistics for EFA and EFA bifactor.

\begin{tabular}{|c|c|c|c|c|c|c|c|}
\hline Model & $\begin{array}{l}\text { Chi-Square } \\
\text { Value }\end{array}$ & $\begin{array}{c}\text { Chi-Square } \\
\text { df }\end{array}$ & Chi-square/df & CFI & TLI & RMSEA & SRMR \\
\hline \multicolumn{8}{|l|}{$\begin{array}{c}\text { Sample } \\
(N=313)\end{array}$} \\
\hline $\begin{array}{l}\text { MLR EFA with } \\
2 \text { factors }\end{array}$ & 77.30 & 26 & 2.97 & .950 & .914 & .079 & .028 \\
\hline $\begin{array}{l}\text { MLR EFA bifactor } \\
\text { with } 2 \text { factors }\end{array}$ & 52.89 & 18 & 2.93 & .966 & .915 & .079 & .023 \\
\hline
\end{tabular}


Table 2. Factor loadings \& factor correlations for the EFA models tested.

\begin{tabular}{cccccc}
\hline & \multicolumn{2}{c}{ Standard EFA } & \multicolumn{3}{c}{ Bifactor EFA } \\
\cline { 2 - 6 }$N=313$ & $\begin{array}{c}\text { Presence } \\
(\mathrm{P})\end{array}$ & Search (S) & General Factor & $\begin{array}{c}\text { Specific P. } \\
\text { Factor }\end{array}$ & $\begin{array}{c}\text { Specific S. } \\
\text { Factor }\end{array}$ \\
\hline Item 1-P & .783 & .122 & $.775^{*}$ & -.006 & .099 \\
Item 2-S & .036 & .699 & .045 & $.0702^{*}$ & .005 \\
Item 3-S & .251 & .824 & $.287^{*}$ & $.788^{*}$ & -.023 \\
Item 4-P & .833 & .129 & $.860^{*}$ & -.014 & .053 \\
Item 5-P & .807 & .087 & $.685^{*}$ & .000 & 1.086 \\
Item 6-P & .800 & .127 & $.741^{*}$ & .007 & .182 \\
Item 7-S & .355 & .704 & $.355^{*}$ & $.651^{*}$ & .047 \\
Item 8-S & .119 & .797 & .141 & $.782^{*}$ & -.027 \\
Item 9-P & .493 & -.189 & $.511^{*}$ & $-.282^{*}$ & .007 \\
Item 10-S & -.219 & .633 & $.230^{*}$ & $.688^{*}$ & .035 \\
Factor & & $.149^{*}$ & & & \\
Correlation & & & & & \\
\hline
\end{tabular}

Bold indicates significant primary loading in EFA and weaker factor loadings for Specific factors in Bifactor EFA. ${ }^{*}$ Significant at $5 \%$ level.

and Search Factor was .149 $(p<.05)$. Steger et al. (2006) reported a weak negative correlation between the two MLQ Factors. In the bifactor solution, all factor loadings were much higher on the general factor than the intended specific group factor, generating the assumption that the MLQ items may measure a higher order construct of meaning. The marginally better fit statistics of the bifactor solution further support this suggestion. However, Bifactor models always tend to support unidimensionality (Joshanloo, Jose, \& Kielpikowski, 2017). Thus, we decided to examine the MLQ factor structure further with Confirmatory Factor Analysis.

\subsection{Confirming Construct Validity with Confirmatory Factor Analysis (CFA)}

MLR was also used to estimate the models in all Confirmatory Factor Analyses. Goodness of fit was evaluated by the Standardized Root Mean Square Residual (SRMR), the Root Mean Square Error of Approximation (RMSEA, 90\% CI), the Comparative Fit Index (CFI), and finally by the Tucker-Lewis index (TLI). Model fit was evaluated by the following criteria (Hu \& Bentler, 1999; Brown, 2015): RMSEA ( $\leq .06,90 \% \mathrm{CI} \leq .06)$, SRMR $(\leq .08)$, CFI $(\geq .95)$, TLI $(\geq .95)$, and the chi-square/df ratio less than 3 (Kline, 2016). Models evaluated were the following: 1) a single-factor Independent Cluster Model of CFA(ICM-CFA) which is generally recommended (Brown, 2015; Crawford \& Henry, 2004) to examine the hypothesis of maximum parsimony; 2) a two-factor ICM-CFA model proposed by Steger at al. (2006). A variation of this model was also tested by adding a covariance between items 2 and 3 and a covariance between items 7 and 8 ; 3) a 
CFA bifactor model (Schmid \& Leiman, 1957, c.f. Reise, 2012), with presence and search in two factors and simultaneously tapping a general factor of life meaning, according to Reise et al. (2007), as quoted in Hammer \& Toland, 2016). A variation of this model was also tested by adding a covariance between items 2 and 3 and a covariance between items 7 and 8; 4) a two-factor Exploratory Structural Equation Model (ESEM; Asparouhov \& Muthen 2009) with all 10 MLQ items loading on the two MLQ factors simultaneously. A variation of this model was also tested by adding a covariance between items 2 and 3 and a covariance between items 7 and 8. ESEM produces more unbiased and accurate models in comparison to ICM-CFA because all secondary loadings are not constrained to zero like in ICM-CFA (Asparouhov \& Muthen, 2009). Therefore, in ESEM models factor correlations are more accurate even with trivial secondary loadings (Howard, Gagne, Morin, Wang \& Forest, 2016).

We did not test a higher order model. For a two first-order factorial structure, like MLQ, evaluating if the second-order factor improves the model fit when compared to the first-order solution is not possible because of under-identification of the higher order model (Wang and Wang, 2012). The fit of all seven alternative CFA models for each sample is presented in Table 3.

Regarding model fit, the single factor ICM-CFA (simple CFA) model performed poorly. The two-factor ICM-CFA model presented almost acceptable fit, both with and without covariances. The bifactor models showed notably better fit than the two-factor models with all fit statistics within acceptable limits (see Table 3). Among them, Bifactor model with covariances showed the best fit to the data. However, the ESEM two-factor models also presented equally adequate fit, with all fit measures notable above fit criteria. Unsurprisingly, ESEM two-factor model with covariances showed better fit than the equivalent non-covariant ESEM model. Consequently, two competing optimal models emerged: 1) the two-factor bifactor with covariances model, and 2) the two-factor ESEM with covariances model (see all fit statistics in Table 3 and Figure 1 for path diagram).

Table 3. Model Fit statistics for the CFA and ESEM models tested with the MLR estimator in the confirmation sample.

\begin{tabular}{lccccccccc}
\hline \multicolumn{1}{c}{ Model } & $\begin{array}{c}\text { Chi-Square } \\
\text { Value }\end{array}$ & $\begin{array}{c}\text { Chi-Square } \\
\text { df }\end{array}$ & Chi-Square/df & CFI & TLI & RMSEA & $\begin{array}{c}\text { RMSEA } \\
\text { Lower CI Higher CI }\end{array}$ & RRMR \\
\hline \multicolumn{1}{c}{ CFA 1 Sample (N = 624) } & & & & & & & & & \\
1) MLR Single Factor ICM-CFA & 1318.22 & 35 & 37.7 & .345 & .157 & .242 & .231 & .254 & .237 \\
2) MLR 2-Factor ICM-CFA & 161.89 & 34 & 4.8 & .935 & .914 & .078 & .066 & .090 & .069 \\
3) MLR 2-Factor ICM-CFAwCov & 134.14 & 33 & 4,1 & .948 & .930 & .070 & .058 & .083 & .068 \\
4) MLR 2-Factor Bifactor & 67.31 & 25 & 2.7 & .978 & .961 & .052 & .037 & .067 & .040 \\
5) MLR 2-Factor BifactorwCov & 45.45 & 24 & 1.9 & .989 & .979 & .038 & .020 & .055 & .040 \\
6) MLR 2-Factor ESEM & 79.58 & 26 & 3.1 & .973 & .953 & .057 & .043 & .072 & .023 \\
7) MLR 2-Factor ESEM with Cov & 51.46 & 23 & 2.2 & .985 & .972 & .045 & .028 & .061 & .019 \\
\hline
\end{tabular}




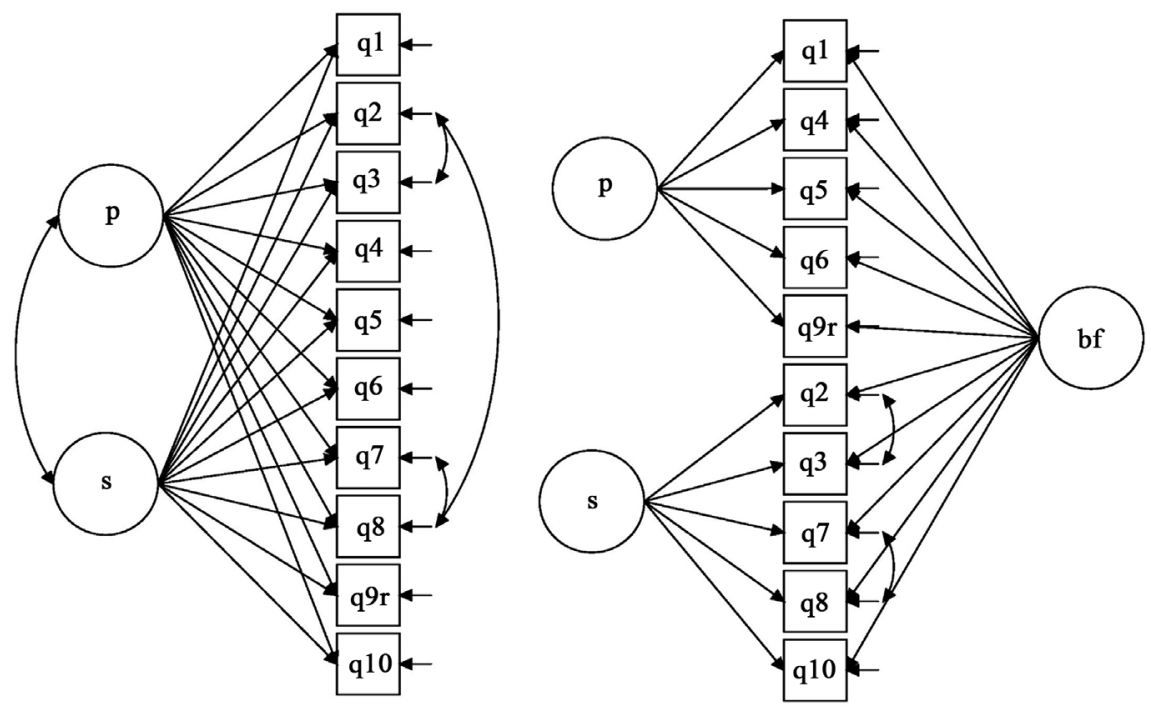

Figure 1. A path diagram of the two optimal models found: ESEM model (Left) and CFA bifactor model (right). Conventionally, latent factors are represented by circles, errors as small arrows pointing on rectangles that represent manifest variables. Single-headed arrows connecting the variables represent a causal path while double-headed arrows on latent variables denote correlation. Double headed arrows between manifest variables denote error covariance.

However, like ICM-CFA methods, Bifactor analysis ignores cross-loadings therefore resulting in a general factor with an overestimated variance (Morin et al., 2016a). Even trivial, unaccounted secondary loadings can inflate factor correlations leading to misspecification (Marsh et al., 2014; Asparouhov \& Muthen 2009; Howard et al., 2016). Therefore, unidimensionality based only on bifactor analysis is often questionable (Joshanloo et al., 2017; Joshanloo \& Jovanovic, 2016). Thus, we suggest that the two-factor ESEM model with covariances be preferable to the bifactor model with covariances (see Figure 1 for more details). Finally, it should be noted that in the ICM-CFA Model tested, the correlation of the Presence with the Search factor was weak but negative $(-0.10)$, in line with Steger et al. (2006).

\subsection{Cross-Validation CFA in a Different Sample}

After determining the optimal model, cross-validation of this model followed (Byrne, 2010; Brown, 2015) to test whether model fit in a different sub-sample of the dataset $(N=624)$. Cross validation of the optimal two-factor ESEM model with covariances was implemented using the MLR estimator (Muthen \& $\mathrm{Mu}$ then, 2012). As shown in Table 4, fit statistics showed an adequate fit to the cross-validation sample with all measures within acceptable limits. Additionally, all fit measures in CFA 2 sample had comparable values with their corresponding fit measures in the model emerged in CFA1 sample (see Table 4 for comparison).

\subsection{Measurement Invariance}

To test for measurement invariance across gender groups, the baseline 
Table 4. Model Fit comparison for the optimal two-factor ESEM model with covariances in a validation and a cross-validation sample.

\begin{tabular}{|c|c|c|c|c|c|c|c|c|c|}
\hline Model & $\begin{array}{l}\text { Chi-Square } \\
\text { Value }\end{array}$ & $\begin{array}{c}\text { Chi-Square } \\
\text { df }\end{array}$ & Chi-square/df & CFI & TLI & RMSEA & $\begin{array}{l}\text { RMSEA } \\
\text { Lower CI }\end{array}$ & $\begin{array}{c}\text { RMSEA } \\
\text { Higher CI }\end{array}$ & SRMR \\
\hline \multicolumn{10}{|l|}{ CFA 1 Sample $(N=624)$} \\
\hline MLR 2 Factor ESEM with Cov & 51.46 & 23 & 2.2 & .985 & .972 & .045 & .028 & .061 & .019 \\
\hline \multicolumn{10}{|l|}{ CFA 2 Sample $(N=624)$} \\
\hline MLR 2 Factor ESEM with Cov & 73.47 & 23 & 3.2 & .985 & .972 & .059 & .044 & .075 & .024 \\
\hline
\end{tabular}

two-factor ESEM model with covariances was tested separately in each gender group (males, $N=599$ vs. females, $N=962$ ). To compare nested models for invariance across gender, we used the $\triangle \mathrm{CFI}$ and $\triangle$ RMSEA criteria proposed by Cheung \& Rensvold (2002). The model fitted the data very well in females (Chi-square $=80.82, p=.0000$, RMSEA $=.051, \mathrm{CFI}=.981)$ and equally well in males (Chi-square $=65.09, p=.0000, \mathrm{RMSEA}=.055, \mathrm{CFI}=.975)$. To evaluate measurement invariance, the model was then tested in both gender groups simultaneously. This model (M1) showed acceptable fit (see Table 5), suggesting that configural invariance was supported. Equality constraints were then imposed on all factor loadings across the two gender groups. As shown in Table 5 both $\triangle$ CFI and $\triangle$ RMSEA in this constrained model (M2) suggested full metric invariance. Finally, all intercepts were constrained to be equal (M3), and both $\triangle \mathrm{CFI}$ and $\triangle$ RMSEA showed full scalar invariance. Thus, we assume that the MLQ is invariant across gender.

\subsection{Internal Consistency Reliability}

Internal consistency reliability of MLQ was evaluated with Cronbach's alpha coefficient (Cronbach, 1951). Values $\geq .70$ are generally considered acceptable and $\geq .80$ adequate (Kline, 2016; Nunnally \& Berstein, 1995; Nunnally, 1978). Alpha coefficient for the total MLQ, the Presence factor and the Search factor were $.76, .85$, and .86 respectively. All results considered together (values from .76 to .86 ) suggest that the scale shows adequate internal reliability.

\subsection{Convergent and Discriminant Validity}

Convergent and discriminant validity was assessed using the total sample ( $N=$ 1561). The Presence of meaning was correlated with search for meaning with a positive, weak and non-significant correlation $(r=.23)$. The correlations of Presence of meaning with measures of well-being were positive and significant ranging from weak $(r=.35, p<.01$, with Hope pathways) to moderate $(r=.53$ with life satisfaction, $p<.01), M=.45$. Similarly, the presence of meaning had positive and significant correlations (all $p s<.01$ ) with the dimensions of CD-RISC, varying from weak ( $r=.23$, Spiritual influences) to moderate ( $r=.55$, control), $M=.39$. As expected, the correlations of presence of meaning with Depression, Anxiety and Stress were all negative and weak but significant $(p s<.01)$, 
Table 5. MLQ Measurement invariance of optimal 2-factor ESEM model across gender.

\begin{tabular}{cccccccc}
\hline$N=1561$ & Chi-Square & Df & CFI & RMSEA & $\begin{array}{c}\text { Model } \\
\text { Comparison }\end{array}$ & $\Delta$ CFI & $\Delta$ RMSEA \\
\hline $\begin{array}{c}\text { M1. } \\
\begin{array}{c}\text { Configural } \\
\text { Invariance }\end{array}\end{array}$ & 145.62 & 46 & .979 & .053 & - & - & - \\
$\begin{array}{c}\text { M2. Full Metric } \\
\text { Invariance }\end{array}$ & 163.63 & 62 & .978 & .046 & M2-M1 & -.001 & -.007 \\
$\begin{array}{c}\text { M3. Full } \\
\text { Scalar Invariance }\end{array}$ & 173.21 & 70 & .978 & .043 & M3-M2 & .000 & -.003 \\
\hline
\end{tabular}

from $r=-.28$ with Stress to $r=-.40$ with Depression, $M=-.33$. Search for meaning had a negative correlation with happiness that was, weak and non-significant. Search for meaning had no correlation with life satisfaction and weak but significant positive correlations with both Agency and Pathways $(r=.12$, $p s<.01)$. The correlations of search for meaning and CD-RISC dimensions were also positive and significant $(p<.01)$ but equally faint, from .07 to $.15, M=.11$. The same was true for Search for meaning and Stress, Anxiety and Depression, with very weak, positive correlations $(M=.06)$ of mixed significance (see Table 6 for details).

\subsection{Descriptive Statistics of MLQ Scores}

In comparison with the scores of the Search factor $(M=23.08, S D=6.99)$, the Presence factor scores were higher and more invariable $(M=25.69, S D=5.73)$. Most participants scored high both on Meaning factor and Search factor $(>24)$, followed by those that scored high in the Presence factor ( $>24)$ but not equally high in the Search factor (see Table 7 for details).

\section{Discussion}

The purpose of this study was to evaluate the following: 1) the construct validity of the Meaning in Life Questionnaire (Steger et al., 2006), Greek Version using different explorative and confirmative factorial analysis approaches like Bifactor EFA, ICM-CFA, Bifactor CFA and ESEM; 2) the measurement invariance of MLQ across gender; 3) the internal consistency reliability of the MLQ; and 4) the convergent and discriminant validity of the MLQ with measures of well-being and mental distress. The results suggested that the data fit the ESEM representation better than both the ICM-CFA model and the bifactor model.

More specifically, the sample was split into three different sub-samples (Guadagnoli \& Velicer, 1988; MacCallum et al., 1996), maintaining enough sample power in each sample to ensure robustness of the models found. Generally, sample-splitting is used as a cross-validation method (Brown, 2015; Byrne, 2010). Regarding sample power, all three samples were far beyond the suggested 10 cases for each observed variable threshold (Osborne \& Costello, 2005; Singh et al., 2016) and larger than 300 or 500 cases (Tabachnick \& Fidell 1996; Comrey \& Lee, 1992), suggested as sufficient sample size for factor analysis. 
Table 6. Bivariate correlation of MLQ on the total sample.

\begin{tabular}{|c|c|c|}
\hline & \multicolumn{2}{|c|}{ Spearman's rho Correlation Coefficient } \\
\hline & MLQ Presence of Meaning & MLQ Search for Meaning \\
\hline \multicolumn{3}{|l|}{ Total Sample $(N=1561)$} \\
\hline MLQ Presence of Meaning & - & .02 \\
\hline MLQ Search for Meaning & .02 & - \\
\hline \multicolumn{3}{|l|}{ Well-Being Dimensions } \\
\hline Subjective Happiness Scale & $.44^{* *}$ & -.03 \\
\hline Satisfaction with Life Scale & $.53^{* *}$ & 0 \\
\hline Trait Hope Scale Agency & $.46^{\star *}$ & $.12^{\star *}$ \\
\hline Trait Hope Scale Pathways & $.35^{\star *}$ & $.12^{* *}$ \\
\hline \multicolumn{3}{|l|}{ Resilience Dimensions } \\
\hline CD-RISC Pers. Competence & $.43^{* *}$ & $.15^{\star *}$ \\
\hline CD-RISC Tolerance/Trust & $.36^{* *}$ & $.12^{* *}$ \\
\hline CD-RISC Secure relat. & $.39^{* *}$ & $.09^{* *}$ \\
\hline CD-RISC Control & $.52^{\star *}$ & $.10^{* *}$ \\
\hline CD-RISC Spir. Influences & $.23^{* *}$ & $.07^{\star *}$ \\
\hline \multicolumn{3}{|l|}{ Mental Distress Dimensions } \\
\hline DASS Stress & $-.28^{\star *}$ & $.08^{* *}$ \\
\hline DASS Anxiety & $-.30^{\star *}$ & $.06^{*}$ \\
\hline DASS Depression & $-.40^{\star *}$ & .05 \\
\hline
\end{tabular}

${ }^{* *} p<.01 ;{ }^{*} p<.05$.

Table 7. Summary statistics for MLQ score for the total sample.

\begin{tabular}{cccc}
\hline & $M$ & $S D$ & Range \\
\hline Sample $(\boldsymbol{N}=\mathbf{1 5 6 1})$ & & & 55 \\
Total MLQ & 48.77 & 8.97 & 29 \\
Presence of meaning factor & 25.69 & 5.73 & 30 \\
Search for meaning factor & 23.08 & 6.99 & 30 \\
\hline
\end{tabular}

First two alternative models were tested using Exploratory factor analysis (EFA): 1) a standard EFA to examine the factor structure and to have a baseline model for EFA comparisons, and 2) a bifactor EFA. Generally, both solutions showed adequate fit to the data, with all fit indices within acceptability criteria and significant factor loadings, all in their intended factor with no exceptions. Generally, the fit of the two models tested was very comparable with the Bifactor model having an overall better fit than the standard EFA model. This may not necessarily suggest a predominant general Meaning-in-life factor be present, since unidimensionality based only on bifactor analysis is unstable (Joshanloo et al., 2017; Joshanloo \& Jovanovic, 2016). 
To examine this assumption further, a Confirmatory factor analysis (CFA) followed in a second sample to verify the models emerged from EFA and bifactor EFA. All CFA models with error covariance were better than their counterparts with no covariance. Note that two covariances added to items of the search factor (see Figure 1) -namely, to item two ("I am looking for something that makes my life feel meaningful") with item three ("I am always looking to find my life's purpose") and to item seven ("I am always searching for something that makes my life feel significant") with eight ("I am seeking a purpose or mission for my life."). Finally, two CFA models showed optimal fit among the seven alternative models tested: 1) The two-factor ESEM model with covariances, and 2) the two-factor bifactor model with covariances. Comparing ESEM to Bi-factor factorial analysis, the latter is a CFA subcategory, allocating the variance of items both into a general factor and sub-factors. Each item is specified to load on the general factor and also its target group factor (Reise, 2012), here "Presence of meaning" and "Search for meaning". Bifactor analysis allows for an examination of the common variance shared by the two MLQ factors and the unique variance specific to each of them.

However, bifactor analysis has received some criticism (Reise et al., 2013; Reise, 2012; Joshanloo et al., 2017). More specifically, it seems that relying solely on the results of bifactor analysis to decide whether a psychological scale is unidimensional or multidimensional may be questionable (Joshanloo et al., 2017). Additionally, constraining non-zero cross-loadings to zero can inflate the variance attributed to the general factor in bifactor analysis (Morin et al., 2016; Joshanloo et al., 2017). Given all above considerations, and the commonness of non-trivial secondary loadings in construct validation, bifactor analysis more often than not is expected to support unidimensionality (Joshanloo \& Jovanovic, 2016). All these limitations considered, we suggested that the two-factor ESEM model with covariances is preferable to the bifactor model.

Additionally, in the CFA, a noteworthy difference was found between this study and previous research. In contrast to this study, RMSE was generally reported to be relatively high in previous research (Damasio \& Koller, 2015; Steger et al., 2006). This improvement in RMSE values in this study in comparison to reported empirical research, could possibly be attributed to ESEM and Bifactor techniques used here.

The MLQ Factors in EFA were positively, weakly correlated at 5\% level of significance. However, the correlation of the Presence with the Search factor in the ICM-CFA model tested was weak and marginally negative. This difference in the relationship of the two factors in EFA and CFA may reflect the mixed influences of both collectivistic and individualistic cultures (Hofstede, 2001; Triandis, 1995) on the Greek sociocultural context, since Greece (along with Cyprus) is situated in the south-eastern border of Europe. This finding is compatible with previous research, suggesting that in collectivistic cultures (e.g., Japan: Steger et al., 2008c or China: Wang \& Dai, 2008) presence of meaning and search for meaning are 
positively correlated. The opposite is true in individualistic cultures (e.g., US: Steger et al., 2006). Equally, ways of finding personal meaning and the relationship between Search and Presence, could be affected by cognitive orientation at individual level (Steger et al., 2008c), and by differences in social orientation across cultures at a group level respectively (Boyraz et al., 2013).

The results of measurement invariance (c.f. Brown, 2015; Byrne, 2010; Kline, 2016; Schumacker \& Lomax, 2015) of the cross-validated model emerged across gender suggested that all 10 items of the MLQ were invariant (full invariance) when used both by males and females. In other words, results from this multi-group CFA suggest that 1) across the two genders, the pattern of fixed and free parameters of the MLQ was equivalent (configural invariance); 2) across gender corresponding factor loadings of the $10 \mathrm{MLQ}$ items were comparable (metric invariance or weak factorial invariance), and 2) across gender corresponding indicator means (intercepts) were equivalent (full scalar invariance or Strong factorial invariance). Furthermore, the results supporting the measurement invariance of the MLQ across gender are adding to previous research findings in several diverse cultures (e.g., Damasio \& Koller, 2015; Boyraz, et al., 2013). According to Damasio \& Koller (2015) invariance suggests a significant quality indicator for the MLQ, enabling valid group comparisons between genders, free from response bias.

Additionally, internal consistency reliability of the Greek MLQ is adequate. Specifically, Cronbach's alpha values were comparable to the results reported by Steger et al. (2006) and by other studies (e.g., Steger \& Samman, 2012; Chan, 2014; Jiang, Bai, \& Xue, 2016; Steger et al., 2008c). Lastly, convergent and discriminant validity of the Greek MLQ was also examined. Expected correlations were found between MLQ and dimensions of well-being, hope, resilience, stress, anxiety and depression. Presence of meaning had significant correlations with all the above measures. The magnitude of the relationships ranged from low to strong. The opposite was true for the Search for meaning, since the significance of the relations was of mixed level and the relationships with the above constructs were weak. The results of the correlation analysis were expected since the MLQ was reported to have overlapping content with other related variables (Steger et al., 2006). Concerning the importance of the relationships, the Presence of meaning factor had different relationships with other constructs tested from MLQ Search for meaning both in magnitude and in direction. This is not surprising, since the two factors of MLQ are reported to have a weak, negative correlation according to Steger et al. (2006).

\section{Conclusion}

Finally, the general conclusion of this work is that the two-factor structure of MLQ established by Steger et al. (2006) is confirmed on the Greek cultural environment because all alternative two-factor models tested by different factorial techniques (Bifactor EFA, ICM CFA, Bifactor CFA and ESEM) showed adequate 
fit to the data. Nevertheless, the optimal model among all different two-factor models tested was the ESEM model with error covariances. Considering all above findings, we reach the conclusion that the Meaning in Life Questionnaire, Greek version is a valid and reliable measure to use in the Greek context. A second important finding of this research is that MLQ Greek is gender equivalent thus, it can be unbiasedly used by both men and women.

This study has certain limitations that should be taken into consideration. First, during the data collection process, trained psychology students were involved. The impact of this method, if any, is unknown. Consequently, any generalization to other populations should be made with caution. Second, missing values in the dataset were estimated with the Expectation-Maximization algorithm (EM). Despite that EM is particularly appropriate for Factor Analysis, the information whether this method assumes a distribution that does not violate the assumption of normality (IBM, 2016) or not (Soley-Bori, 2013) is unclear.

Moreover, error covariances used in optimal model possibly suggest an overlapping content of the items (Brown, 2015). A similar issue was reported by Damasio \& Koller (2005) for the Brazil version of the MLQ. So, further research is necessary to examine this issue in yet another sample evaluating if it is a culture-specific effect. Future research could also evaluate new confirmatory factor analysis techniques like Bifactor ESEM. Finally, invariance of the MLQ across age is another possible direction of the research in the future. Besides, construct validity is built over time. So, multiple studies should be carried out over different samples to shape more robust evidence of construct validity progressively (Crocker \& Algina, 1986; Messick, 1995).

Despite the above limitations, the contribution of the present study is that we have provided strong evidence for the construct validity, measurement invariance across gender, reliability and convergent/discriminant validity of the MLQ, Greek version. With the use of EFA bifactor, ICM-CFA, CFA Bifactor and ESEM factorial analysis techniques the MLQ Greek is confirmed to be a valid, reliable and gender equivalent measure of well-being.

\section{References}

Adler, M. G., \& Fagley, N. S. (2005). Appreciation: Individual Differences in Finding Value and Meaning as a Unique Predictor of Subjective Well-Being. Journal of Personality, 73, 79-114.

Alfonso, Y. C., \& Allison, D. B. (1992a). Further Development of the Extended Satisfaction with Life Scale. The Behavior Therapist, 5, 15-16.

Antonovsky, A. (1987). Unraveling the Mystery of Health: How People Manage Stress and Stay Well. San Francisco, CA: Jossey-Bass.

Arrindell, W. A., Meeuwesen, L., \& Huyse, F. J. (1991). The Satisfaction with Life Scale (SWLS): Psychometric Properties in a Non-Psychiatric Medical Outpatients Sample. Personality and Individual Differences, 12, 117-123. https://doi.org/10.1016/0191-8869(91)90094-R

Asparouhov, T., \& Muthen, B. (2009). Exploratory Structural Equation Modeling. Struc- 
tural Equation Modeling, 16, 397-438. https://doi.org/10.1080/10705510903008204

Battista, J., \& Almond, R. (1973). The Development of Meaning in Life. Psychiatry, 36, 409-427. https://doi.org/10.1080/00332747.1973.11023774

Bentler, P. M., \& Yuan, K. H. (1999). Structural Equation Modeling with Small Samples: Test of Global Self-Esteem. Journal of Personality and Social Psychology, 89, 623-642.

Blais, M. R., Vallerand, R. J., Palletier, L. G., \& Briere, N. M. (1989). L' Echelle de satisfaction de vie: Validation Canadienne-Francaise du 'Satisfaction with Life Scale'. [French-Canadian Validation of the Satisfaction with Life Scale]. Canadian Journal of Behavioral Science, 21, 210-223.

Boyle, P. A., Buchman, A. S., Barnes, L. L., \& Bennett, D. A. (2010). Effect of a Purpose in Life on Risk of Incident Alzheimer Disease and Mild Cognitive Impairment in Community-Dwelling Older Persons. Archives of General Psychiatry, 67, 304-310. https://doi.org/10.1001/archgenpsychiatry.2009.208

Boyraz, G., \& Efstathiou, N. (2011). Self-Focused Attention, Meaning, and Posttraumatic Growth: The Mediating Role of Positive and Negative Affect for Bereaved Women. Journal of Loss and Trauma, 16, 13-32. https://doi.org/10.1080/15325024.2010.507658

Boyraz, G., Horne, S. G., \& Sayger, T. V. (2010). Finding Positive Meaning after Loss: The Mediating Role of Reflection for Bereaved Individuals. Journal of Loss and Trauma, 15, 242-258. https://doi.org/10.1080/15325020903381683

Boyraz, G., Lightsey Jr., O. R., \& Can, A. (2013). The Turkish Version of the Meaning in Life Questionnaire: Assessing the Measurement Invariance across Turkish and American Adult Samples. Journal of Personality Assessment, 95, 423-431. https://doi.org/10.1080/00223891.2013.765882

Brandstätter, M., Baumann, U., Borasio, G. D., \& Fegg, M. J. (2012). Systematic Review of Meaning in Life Instruments. Psychooncology, 21, 1034-1052.

https://doi.org/10.1002/pon.2113

Bronk, C., Hill, K., Lapsley, P. L., Talib, D. K., Tasneem, L., \& Holmes, F. (2009). Purpose, Hope, and Life Satisfaction in Three Age Groups. The Journal of Positive Psychology, 4, 500-510.

Brown, T. A. (2015). Confirmatory Factor Analysis for Applied Research (2nd ed.). New York, NY: Guilford Publications.

Byrne, B. M. (2010). Structural Equation Modeling with AMOS: Basic Concepts, Applications, and Programming (2nd ed.). New York, NY: Routledge.

Chan, W. C. H. (2014). Factor Structure of the Chinese Version of the Meaning in Life Questionnaire in Hong Kong Chinese Caregivers. Health and Social Work, 393, 135-143. https://doi.org/10.1093/hsw/hlu025

Chan, W. C. H., \& Chan, C. L. W. (2011). Acceptance of Spousal Death: The Factor of Time in Bereaved Older Adults' Search for Meaning. Death Studies, 35, 147-162. https://doi.org/10.1080/07481187.2010.535387

Cheung, G. W., \& Rensvold, R. B. (2002). Evaluating Goodness-of-Fit Indexes for Testing Measurement Invariance. Structural Equation Modeling, 9, 233-255.

Compton, W. C., Smith, M. L., Cornish, K. A., \& Qualls, D. L. (1996). Factor Structure of Mental Health Measures. Journal of Personality and Social Psychology, 71, 406-413. https://doi.org/10.1037/0022-3514.71.2.406

Comrey, A. L., \& Lee, H. B. (1992). A First Course in Factor Analysis (2nd ed.). Hillsdale, NJ: Erlbaum.

Connor, K. M., \& Davidson, J. R. (2003). Development of a New Resilience Scale: The Connor-Davidson Resilience Scale (CD-RISC). Depression and Anxiety, 18, 76-82. 
https://doi.org/10.1002/da.10113

Crawford, J. R., \& Henry, J. D. (2004). The Positive and Negative Affect Schedule (PANAS): Construct Validity, Measurement Properties and Normative Data in a Large Non-Clinical Sample. British Journal of Clinical Psychology, 43, 245-265. https://doi.org/10.1348/0144665031752934

Crocker, L., \& Algina, J. (1986). Introduction to Classical and Modern Test Theory. Orlando, FL: Harcourt Brace Jovanovich.

Cronbach, L. J. (1951). Coefficient Alpha and the Internal Structure of Tests. Psychometrika, 16, 297-334. https://doi.org/10.1007/BF02310555

Crumbaugh, J. C. (1977). The Seeking of Noetic Goals Test (SONG): A Complementary Scale to the Purpose in Life Test (PIL). Journal of Clinical Psychology, 33, 900-907. https://doi.org/10.1002/1097-4679(197707)33:3<900::AID-JCLP2270330362>3.0.CO;2-8

Crumbaugh, J. C., \& Maholick, L. T. (1964). An Experimental Study in Existentialism: The Psychometric Approach to Frankl's Concept of Noogenic Neurosis. Journal of Clinical Psychology, 20, 200-207.

https://doi.org/10.1002/1097-4679(196404)20:2<200::AID-JCLP2270200203>3.0.CO;2-U

Damasio, B., \& Koller, S. H. (2015). Meaning in Life Questionnaire: Adaptation Process and Psychometric Properties of the Brazilian Version. RevistaLatinoamericana de Psicología, 47, 185-195. https://doi.org/10.1016/j.rlp.2015.06.004

Debats, D. L., van der Lubbe, P. M., \& Wezeman, F. R. A. (1993). On the Psychometric Properties of the Life Regard Index (LRI): A Measure of Meaningful Life. Personality and Individual Differences, 14, 337-345.

https://doi.org/10.1016/0191-8869(93)90132-M

Diener, E., \& Seligman, M. E. P. (2004). Beyond Money: Toward an Economy of Well-Being. Psychological Science in the Public Interest, 5, 1-31.

https://doi.org/10.1111/j.0963-7214.2004.00501001.x

Diener, E., Emmons, R. A., Larsen, R. J., \& Griffin, S. (1985). The Satisfaction with Life Scale. Journal of Personality Assessment, 49, 71-75. https://doi.org/10.1207/s15327752jpa4901_13

Doornik, J. A., \& Hansen, H. (2008). An Omnibus Test for Univariate and Multivariate Normality. Oxford Bulletin of Economics and Statistics, 70, 927-939. https://doi.org/10.1111/j.1468-0084.2008.00537.x

Dyck, M. J. (1987). Assessing Logotherapeutic Constructs: Conceptual and Psychometric Status of the Purpose in Life and Seeking of Noetic Goals Tests. Clinical Psychology Review, 7, 439-447. https://doi.org/10.1016/0272-7358(87)90021-3

Frankl, V. E. (1963). Man's Search for Meaning: An Introduction to Logotherapy. New York, NY: Washington Square Press.

Frazier, P., Oishi, S., \& Steger, M. (2003). Assessing Optimal Human Functioning. In W. B. Walsh (Ed.), Contemporary Topics in Vocational Psychology, Counseling Psychology and Optimal Human Functioning (pp. 251-278). Mahwah, NJ: Erlbaum.

Guadagnoli, E., \& Velicer, W. F. (1988). Relation to Sample Size to the Stability of Component Patterns. Psychological Bulletin, 103, 265.

https://doi.org/10.1037/0033-2909.103.2.265

Hammer, J. H., \& Toland, M. D. (2016). Bifactor Analysis in Mplus. http://sites.education.uky.edu/apslab/upcoming-events/

Harlow, L. L., Newcomb, M. D., \& Bentler, P. M. (1986). Depression, Self-Derogation, Substance Use, and Suicide Ideation: Lack of Purpose in Life as a Mediational Factor. Journal of Clinical Psychology, 42, 5-21. 
https://doi.org/10.1002/1097-4679(198601)42:1<5::AID-JCLP2270420102>3.0.CO;2-9

Henry, J., \& Crawford, J. (2005). The Short-Form Version of the Depression Anxiety Stress Scales (DASS-21): Construct Validity and Normative Data in a Large Non-Clinical Sample. The British Journal of Clinical Psychology, 44, 227-239. https://doi.org/10.1348/014466505X29657

Henze, N., \& Zirkler, B. (1990). A Class of Invariant Consistent Tests for Multivariate Normality. Communications in Statistics, Theory and Methods, 19, 3595-3617. https://doi.org/10.1080/03610929008830400

Hofstede, G. (2001). Culture's Consequences: Comparing Values, Behaviors, Institutions, and Organizations across Nations (2nd ed.). Thousand Oaks, CA: Sage.

Howard, J., Gagné, M., Morin, A. J. S., Wang, Z. N., \& Forest, J. (2016). Using Bifactor Exploratory Structural Equation Modeling to Test for a Continuum Structure of Motivation. Journal of Management, 1-59.

Hu, L., \& Bentler, P. M. (1999). Cutoff Criteria for Fit Indexes in Covariance Structure Analysis: Conventional Criteria versus New Alternatives. Structural Equation Modeling, 6, 1-55. https://doi.org/10.1080/10705519909540118

IBM Corporation (2016). IBM SPSS Missing Values 24. In IBM SPSS Statistics Version 24, Release 0 Manual. Spring House, PA: IBM Corporation.

IBM Corporation (2017). IBM SPSS Statistical Software, Release 25. Spring House, PA: IBM Corporation.

Jennrich, R. I., \& Bentler, P. M. (2011). Exploratory Bi-Factor Analysis. Psychometrika, 76, 537-549. https://doi.org/10.1007/s11336-011-9218-4

Jiang, Y., Bai, L., \& Xue, S. (2016). Validation of the Meaning in Life Questionnaire (MLQ) in Chinese University Students and Invariance across Gender. International Journal of Humanities Social Sciences and Education, 3, 41-48.

Joshanloo, M., \& Jovanovic, V. (2016). The Factor Structure of the Mental Health Continuum-Short Form (MHC-SF) in Serbia: An Evaluation using Exploratory Structural Equation Modeling. Journal of Mental Health, 26, 510-515. https://doi.org/10.1080/09638237.2016.1222058

Joshanloo, M., Jose, P. E., \& Kielpikowski, M. (2017). The Value of Exploratory Structural Equation Modeling in Identifying Factor Overlap in the Mental Health Continuum-Short Form (MHC-SF): A Study with a New Zealand Sample. Journal of Happiness Studies, 8, 1061-1074. https://doi.org/10.1007/s10902-016-9767-4

Kiang, L., \& Fuligni, A. J. (2010). Meaning in Life as a Mediator of Ethnic Identity and Adjustment among Adolescents from Latin, Asian, and European American Backgrounds. Journal of Youth and Adolescence, 3, 1253-1264. https://doi.org/10.1007/s10964-009-9475-z

King, L. A., Hicks J. A., Krull J. L., \& Del Gaiso, A. K. (2006). Positive Affect and the Experience of Meaning in Life. Journal of Personality and Social Psychology, 90, 179-196. https://doi.org/10.1037/0022-3514.90.1.179

Kline, R. B. (2016). Principles and Practice of Structural Equation Modeling (4th ed.). New York, NY: The Guilford Press.

Larsen, R. J., Diener, E., \& Emmons, R. A. (1985). An Evaluation of Subjective Well-Being Measures. Social Indicators Research, 17, 1-18. https://doi.org/10.1007/BF00354108

Lee, V. (2008). The Existential Plight of Cancer: Meaning Making as a Concrete Approach to the Intangible Search for Meaning. Supportive Care in Cancer, 16, 779-785. https://doi.org/10.1007/s00520-007-0396-7

Lent, R. W. (2004). Toward a Unifying Theoretical and Practical Perspective on 
Well-Being and Psychosocial Adjustment. Journal of Counseling Psychology, 51, 482-509. https://doi.org/10.1037/0022-0167.51.4.482

Little, R. J. A. (1988). A Test of Missing Completely at Random for Multivariate Data with Missing Values. Journal of the American Statistical Association, 83, 1198-1202.

Lovibond, S. H., \& Lovibond, P. F. (1995). Manual for the Depression Anxiety Stress Scales (DASS). New South Wales: Psychology Foundation Monograph.

Lyubomirsky, S., \& Lepper, H. (1999) A Measure of Subjective Happiness: Preliminary Reliability and Construct Validation. Social Indicators Research, 46, 137-155. https://doi.org/10.1023/A:1006824100041

MacCallum, R. C., Browne, M. W., \& Sugawara, H. M. (1996). Power Analysis and Determination of Sample Size for Covariance Structure Modeling. Psychological Methods, 1, 130. https://doi.org/10.1037/1082-989X.1.2.130

Mardia, K. V. (1970). Measures of Multivariate Skewness and Kurtosis with Applications. Biometrika, 57, 519-530. https://doi.org/10.1093/biomet/57.3.519

Marsh, H. W., Morin, A. J. S., Parker, P. D., \& Kaur, G. (2014). Exploratory Structural Equation Modeling: An Integration of the Best Features of Exploratory and Confirmatory Factor Analysis. Annual Review of Clinical Psychology, 10, 85-110. https://doi.org/10.1146/annurev-clinpsy-032813-153700

Massey, F. J. (1951). The Kolmogorov-Smirnov Tests for Goodness of Fit. Journal of American Statistical Association, 46, 68-78. https://doi.org/10.1080/01621459.1951.10500769

McDowell, I. (2006). Measuring Health: A Guide to Rating Scales and Questionnaires (3rd ed.). New York, NY: Oxford University Press. https://doi.org/10.1093/acprof:oso/9780195165678.001.0001

Messick, S. (1995). Validity of Psychological Assessment: Validation of Inferences from Person's Responses and Performances as Scientific Inquiry into Score Meaning. American Psychologist, 50, 741-749. https://doi.org/10.1037/0003-066X.50.9.741

Morin, A. J. S., Arens, A., \& Marsh, H. (2016a). A Bifactor Exploratory Structural Equation Modeling Framework for the Identification of Distinct Sources of Construct-Relevant Psychometric Multidimensionality. Structural Equation Modeling, 23, 116-139. https://doi.org/10.1080/10705511.2014.961800

Muthen, B., \& Asparouhov, T. (2002). Using Mplus Monte Carlo Simulations in Practice: A Note on Non-Normal Missing Data in Latent Variable Models. Mplus Web Notes: No. 2.

Muthen, L. K., \& Muthen, B. O. (2012). Mplus Statistical Software, 1998-2012. Los Angeles, CA: Author.

Nunnally, J. C. (1978). Psychometric Theory(2nd ed.). New York, NY: McGraw-Hill.

Nunnally, J., \& Berstein, I. (1995). Psychometric Theory. New York, NY: McGraw-Hill.

Osborne, J. W., \& Costello, A. B. (2005). Sample Size and Subject to Item Ratio in Principal Components Analysis. Practical Assessment, Research \& Evaluation, 9, 8.

Park, N., Park, M., \& Peterson, C. (2010). When Is Search for Meaning Related to Life Satisfaction? Applied Psychology: Health and Wellbeing, 2, 1-13. https://doi.org/10.1111/j.1758-0854.2009.01024.x

Pavot, W., \& Diener, E. (1993). The Affective and Cognitive Context of Self-Reported Measures of Subjective Well-Being. Social Indicators Research, 28, 1-20.

https://doi.org/10.1007/BF01086714

Pavot, W., \& Diener, E. (2008). The Satisfaction with Life Scale and the Emerging Construct of Life Satisfaction. The Journal of Positive Psychology, 3, 137-152. 
https://doi.org/10.1080/17439760701756946

Pezirkianidis, C., Galanakis, M., Karakasidou, I., \& Stalikas, A. (2016). Validation of the Meaning in Life Questionnaire (MLQ) in a Greek Sample. Psychology, 7, 1518-1530. https://doi.org/10.4236/psych.2016.713148

Reise, S. (2012). The Rediscovery of Bifactor Measurement Models. Multivariate Behavioral Research, 47, 667-696. https://doi.org/10.1080/00273171.2012.715555

Reise, S. P., Morizot, J., \& Hays, R. D. (2007). The Role of the Bifactor Model in Resolving Dimensionality Issues in Health Outcomes Measures. Quality of Life Research, 16, 19-31.

Reise, S. P., Scheines, R., Widaman, K. F., \& Haviland, M. G. (2013). Multidimensionality and Structural Coefficient Bias in Structural Equation Modeling: A Bifactor Perspective. Educational and Psychological Measurement, 73, 5-26. https://doi.org/10.1177/0013164412449831

Reker, G. T., Peacock, E. J., \& Wong, P. T. P. (1987). Meaning and Purpose in Life and Well-Being: A Life-Span Perspective. Journal of Gerontology, 42, 44-49. https://doi.org/10.1093/geronj/42.1.44

Ryan, R. M., \& Deci, E. L. (2001). On Happiness and Human Potentials: A Review of Research on Hedonic and Eudaimonic Well-Being. Annual Review of Psychology, 52, 141-166. https://doi.org/10.1146/annurev.psych.52.1.141

Ryff, C. D. (1989). Happiness Is Everything, or Is It? Explorations on the Meaning of Psychological Well-Being. Journal of Personality and Social Psychology, 57, 1069-1081. https://doi.org/10.1037/0022-3514.57.6.1069

Ryff, C. D., \& Singer, B. H. (1998). The Contours of Positive Human Health. Psychological Inquiry, 9, 1-28. https://doi.org/10.1207/s15327965pli0901_1

Schmid, J., \& Leiman, J. N. (1957). The Development of Hierarchical Factor Solutions. Psychometrika, 22, 53-61. https://doi.org/10.1007/BF02289209

Schulenberg, S. E., Strack, K. M., \& Buchanan, E. M. (2011). The Meaning in Life Questionnaire: Psychometric Properties with Individuals with Serious Mental Illness in an Inpatient Setting. Journal of Clinical Psychology, 67, 1210-1219.

https://doi.org/10.1002/jclp.20841

Schumacker, R. E., \& Lomax, R. G. (2015). A Beginner's Guide to Structural Equation Modeling (4th ed.). New York, NY: Routledge.

Seligman, M. E. P., \& Csikszentmihalyi, M. (2000). Positive Psychology: An Introduction. American Psychologist, 55, 5-14. https://doi.org/10.1037/0003-066X.55.1.5

Seligman, M. E. P., Steen, T. A., Park, N., \& Peterson, C. (2005). Positive Psychology Practice: Empirical Validation of Interventions. American Psychologist, 60, 410-421. https://doi.org/10.1037/0003-066X.60.5.410

Singh, K., Junnarkar, M., \& Kaur, J. (2016). Measures of Positive Psychology, Development and Validation. Berlin: Springer. https://doi.org/10.1007/978-81-322-3631-3

Skaggs, B. G., \& Barron, C. (2006). Searching for Meaning in Negative Events: A Concept Analysis. Journal of Advanced Nursing, 53, 559-570. https://doi.org/10.1111/j.1365-2648.2006.03761.x

Snyder, C. R., Harris, C., Anderson, J. R., Holleran, S. A., Irving, L. M., Sigmon, S. T., Yoshinobu, L., Gibb, J., Langelle, C., \& Harney, P. (1991). The Will and the Ways: Development and Validation of an Individual-Differences Measure of Hope. Journal of Personality and Social Psychology, 60, 570-585.

https://doi.org/10.1037/0022-3514.60.4.570

Soley-Bori, M. (2013). Dealing with Missing Data: Key Assumptions and Methods for Applied Analysis. Technical Report No. 4, Boston, MA: School of Public Health, Bos- 
ton University.

StataCorp. (2015). Stata: Release 14. Statistical Software. College Station, TX: StataCorp.

Steger, F. M., \& Samman, E. (2012). Assessing Meaning in Life on an International Scale: Psychometric Evidence for the Meaning in Life Questionnaire-Short Form among Chilean Households. International Journal of Wellbeing, 2, 182-195.

https://doi.org/10.5502/ijw.v2i.i3.2

Steger, M. F. (2007). Structural Validity of the Life Regards Index. Measurement and Evaluation in Counseling and Development, 40, 97-109. https://doi.org/10.1080/07481756.2007.11909808

Steger, M. F. (2010). The Meaning in Life Questionnaire (MLQ). http://michaelfsteger.com/

Steger, M. F. (2012). Experiencing Meaning in Life: Optimal Functioning at the Nexus of Spirituality, Psychopathology, and Well-Being. In P. T. P. Wong, \& P. S. Fry (Eds.), The Human Quest for Meaning (2nd ed., pp. 165-184). New York, NY: Routledge.

Steger, M. F., \& Frazier, P. (2005). Meaning in Life: One Link in the Chain from Religiousness to Well-Being. Journal of Counseling Psychology, 52, 574-582. https://doi.org/10.1037/0022-0167.52.4.574

Steger, M. F., \& Kashdan, T. B. (2006). Stability and Specificity of Meaning in Life and Life Satisfaction over One Year: Implications for Outcome Assessment. Journal of Happiness Studies, 8, 161-179. https://doi.org/10.1007/s10902-006-9011-8

Steger, M. F., \& Shin, J. Y. (2010). The Relevance of the Meaning in Life Questionnaire to Therapeutic Practice: A Look at the Initial Evidence. The International Forum for Logotherapy, 33, 95-104.

Steger, M. F., Frazier, P., \& Zacchanini, J. L. (2008a). Terrorism in Two Cultures: Traumatization and Existential Protective Factors Following the September 11th Attacks and the Madrid train Bombings. Journal of Trauma and Loss, 13, 511-527. https://doi.org/10.1080/15325020802173660

Steger, M. F., Frazier, P., Oishi, S., \& Kaler, M. (2006). The Meaning in Life Questionnaire. Assessing the Presence of and Search for Meaning in Life. Journal of Counseling Psychology, 53, 80-93. https://doi.org/10.1037/0022-0167.53.1.80

Steger, M. F., Kashdan, T. B., Sullivan, B. A., \& Lorentz, D. (2008b). Understanding the Search for Meaning in Life: Personality, Cognitive Style, and the Dynamic between Seeking and Experiencing Meaning. Journal of Personality, 76, 199-228. https://doi.org/10.1111/j.1467-6494.2007.00484.x

Steger, M. F., Kawabata, Y., Shimai, S., \& Otake, K. (2008c). The Meaningful Life in Japan and the United States: Levels and Correlates of Meaning in Life. Journal of Research in Personality, 42, 660-678. https://doi.org/10.1016/j.jrp.2007.09.003

Steger, M. F., Mann, J. R., Michels, P., \& Cooper, T. C. (2009). Meaning in Life, Anxiety, Depression, and General Health among Smoking Cessation Patients. Journal of Psychosomatic Research, 67, 353-358. https://doi.org/10.1016/j.jpsychores.2009.02.006

Tabachnick, B. G., \& Fidell, L. S. (1996). Using Multivariate Statistics (3rd ed.). New York, NY: HarperCollins.

Temane, L., Khumalo, I. P., \& Wissing, M. P. (2014). Validation of the Meaning in Life Questionnaire in a South African Context. Journal of Psychology in Africa, 24, 81-95.

Triandis, H. C. (1995). Individualism and Collectivism. Boulder, CO: Westview.

Wang, J., \& Wang, X. (2012). Structural Equation Modeling. Hoboken, NJ: Wiley, Higher Education Press. https://doi.org/10.1002/9781118356258 
Wang, M. C., \& Dai, X. Y. (2008). Chinese Meaning in Life Questionnaire Revised in College Students and Its Reliability and Validity Test. Chinese Journal of Clinical Psychology, 16, 459-461.

Yusoff, M. S. B. (2013). Psychometric Properties of the Depression Anxiety Stress Scale in a Sample of Medical Degree Applicants. International Medical Journal, 20, 295-300.

Zika, S., \& Chamberlain, K. (1992). On the Relation between Meaning in Life and Psychological Well-Being. British Journal of Psychology, 83, 133-145.

https://doi.org/10.1111/j.2044-8295.1992.tb02429.x 